\title{
Changing Paradigms of Treating Severe Keratitis in Dry Eye Disease
}

\author{
Proceedings of a Symposium Presented at the European Society of Ophthalmology 2015 Congress in \\ Vienna, Austria on 7 June 2015
}

\author{
Symposium Speakers: Christophe Baudouin, ${ }^{1}$ Mourad Amrane, ${ }^{2}$ Andrea Leonardi ${ }^{3}$ and Gysbert van Setten ${ }^{4}$ \\ 1. Quinze-Vingts National Ophthalmology Hospital, Paris, France; 2. Santen SAS, Evry, France; \\ 3. Department of Neuroscience, Ophthalmology, University of Padua, Italy; 4. St Eriks Eye Hospital, Stockholm, sweden
}

\begin{abstract}
These proceedings are based on a symposium, presented at the European Society of Ophthalmology 2015 Congress in Vienna, Austria on 8 June 2015. The symposium was arranged by Santen to bring together leaders in ophthalmology with the objective of exploring advances in the treatment of severe keratitis in dry eye disease (DED). DED is a multifactorial disease of the tears and ocular surface that results in symptoms of discomfort, visual disturbance and tear instability with potential damage to the ocular surface. Abnormalities at the ocular surface can affect all tear dynamic components, resulting in a vicious cycle that reinforces/sustains DED. Ciclosporin (CSA) is thought to exert an anti-inflammatory effect on ocular surface cells. This compound should be in the near future one of the principal advances in the treatment of DED for blocking the vicious cycle of the disease. Extensive formulation development work has led to the availability of a well-tolerated, stable cationic nanoemulsion, designated as Novasorb ${ }^{\circledR}$ technology. This technology has been used in the first European approved CSA $1 \mathrm{mg} / \mathrm{mL}$ eye drops emulsion (IKervis ${ }^{\circledR}$ ) for the treatment of severe keratitis in adult patients with DED, which has not improved despite treatment with tear substitutes. Clinical trials involving over 900 patients have demonstrated a good safety and tolerability profile and significant clinical efficacy of this formulation with a once-daily dosing regimen. CsA $1 \mathrm{mg} / \mathrm{mL}$ eye drops were evaluated in the pivotal phase III SANSIKA study, which confirmed the positive benefit-risk profile of CSA $1 \mathrm{mg} / \mathrm{mL}$ eye drops for the treatment of severe keratitis in DED. An assessment of ocular surface health requires: listening to the patient, the use of simple diagnostic tools, an expertise in examination, including use of microscopy, and an up-to-date knowledge of current models of DED.
\end{abstract}

\section{Keywords}

Dry eye disease (DED), ciclosporin, Ikervis ${ }^{\circledR}$, Novasorb ${ }^{\circledR}$ technology, corneal fluorescein staining (CFS), keratitis, inflammation, SANSIKA

Disclosures: Christophe Baudouin is a consultant and has received research grants from Alcon, Allergan, Santen and Thea. Mourad Amrane is an employee of Santen. Andrea Leonardi is a consultant for Alcon, Allergan, Santen, Thea and SIFI. Gysbert van Setten is a consultant to Santen.

Acknowledgements: Editorial assistance was provided by Catherine Amey at Touch Medical Media and funded by Santen.

open Access: This article is published under the Creative Commons Attribution Noncommercial License, which permits any non-commercial use, distribution, adaptation and reproduction provided the original author(s) and source are given appropriate credit.

Received: 10 September 2015 Citation: European Ophthalmic Review, 2015;9(2):121-7

Correspondence: Christophe Baudouin, Quinze-Vingts National Ophthalmology Hospital, 28 Rue de Charenton, 75012 Paris, France. E: cbaudouin@quinze-vingts.fr

Support: The symposia proceedings, and the symposium itself, were produced and paid for by Santen. The views and opinions expressed are those of the authors and not necessarily those of Santen. This article reports the proceedings of a sponsored satellite symposium held at the European Society of Ophthalmology 2015 Congress and, as such, has not been subject to this journal's usual peer-review process. The report was reviewed for scientific accuracy by the symposium speakers before publication.

\section{Rationale for Anti-inflammatory Treatment in Severe Dry Eye}

\author{
Christophe Baudouin
}

Quinze-Vingts National Ophthalmology Hospital, Paris, France

The Dry Eye Workshop, in 2007, defined dry eye disease (DED) as a multifactorial disease of the tears and ocular surface that results in symptoms of discomfort, visual disturbance and tear film instability, with potential damage to the ocular surface. ${ }^{1}$ The definition adds that: "It is accompanied by increased osmolarity of the tear film and inflammation of the ocular surface". Tear dynamics depend on complex interplays between the environment, the eyelids, lacrimal film, conjunctiva and cornea. There are multiple manifestations of DED, both aqueous deficient and evaporative classifications., ${ }^{2,3}$ Aqueous deficient DED includes Sjögren's syndrome (primary and secondary), nonSjögren's dry eye, lacrimal gland duct obstruction, reflex hyposecretion and the use of systemic drugs, such as anti-histamines, beta-blockers, antispasmodics, diuretics and some psychotropic drugs. Evaporative DED may be divided into intrinsic and extrinsic conditions. Intrinsic factors encompass Meibomian gland dysfunction, disorders of the lid aperture and a low blink rate behaviour, whereas vitamin A deficiency, 


\title{
Figure 1: Main Mechanisms of Dry Eye Disease
}

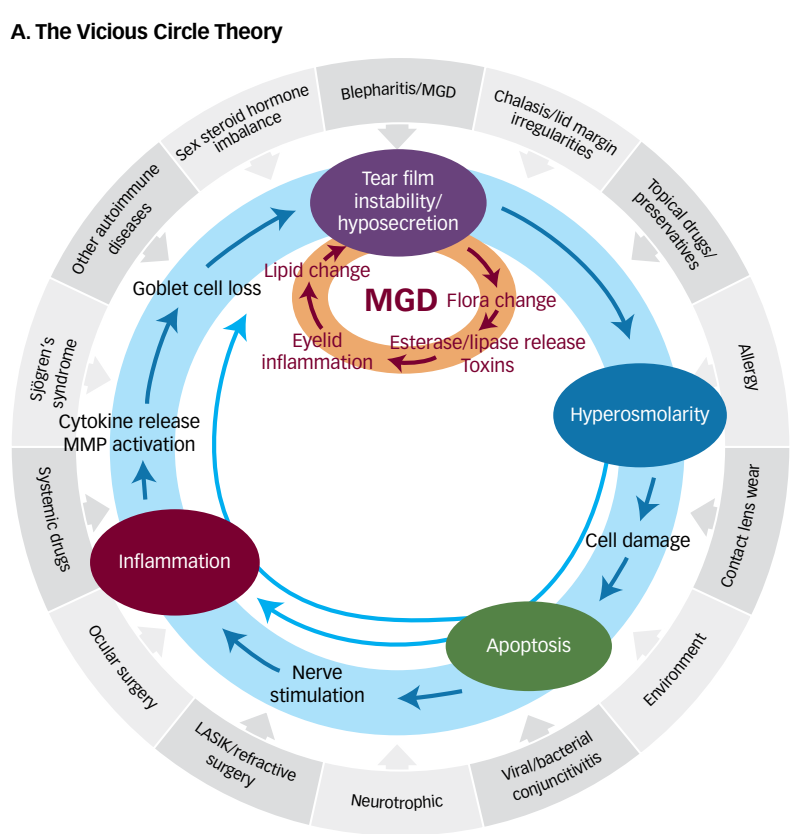

B. Different Therapies Act on Different Points in the Circle

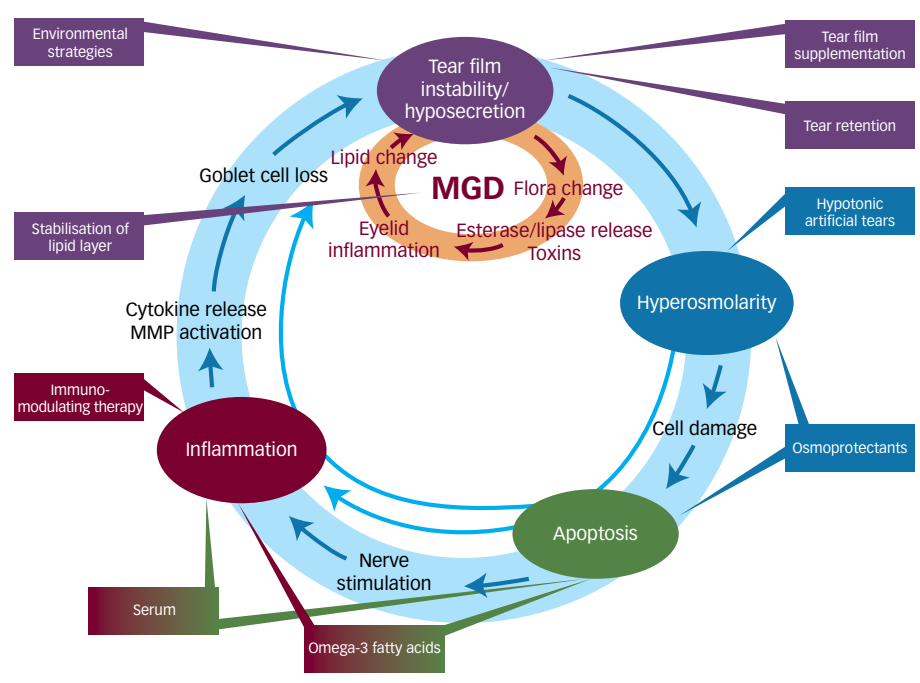

LASIK = laser-assisted in situ keratomileusis; MGD = meibomian gland dysfunction; MMP = matrix metalloproteinase. Adapted from: Baudouin et al., 2007. ${ }^{11}$

topical preservative drugs, contact lens wear and ocular surface disease, e.g. allergy, are all extrinsic factors. ${ }^{4}$

The role of the nervous system in DED has been documented. ${ }^{5}$ The tear film, lacrimal glands, corneal and conjunctival epithelia and Meibomian glands work together as an integrated lacrimal functional unit (LFU). The integrity of the LFU is necessary for the health and normal function of the eye and visual system. Nervous connections and systemic hormones are well-known factors that maintain the homeostasis of the ocular surface. DED has also been hypothesised to be a localised autoimmune disease originating from an imbalance in the protective immunoregulatory and proinflammatory pathways of the ocular surface. ${ }^{6}$ Thus, DED is not restricted to the ocular surface.

The major histocompatibility complex class II cell surface receptor, human leucocyte antigen-D related (HLA-DR) is a good biomarker of severity of inflammation. Other techniques adapted to assessing inflammation in tears include tear proteomics ${ }^{7,8}$ and enzyme-linked immunosorbent assay (ELISA) ${ }^{9}$ to monitor levels of interleukin (IL)-4, IL-5 and tumour necrosis factor-alpha that were found to be raised in atopic keratoconjunctivitis (AKC) patients compared with normal subjects. Another tear assay technique is the multiplex bead analysis. ${ }^{10}$ This can be used to determine IL-1-receptor antagonist (IL-1Ra), IL-6, IL-8/CXCL8 and epidermal growth factor (EGF), which are elevated in DED patients and correlated with DED criteria.

Any abnormality at the ocular surface can initiate disequilibrium in all other components of tear dynamics, resulting in a series of events constituting a vicious circle that reinforces or sustaining DED (see Figure 1A). ${ }^{11}$ Different therapies act at various points in this vicious circle to inhibit its perpetuation (see Figure $1 B$ ). Agents that have been used to block inflammation include off-label use of steroids, ${ }^{12}$ which are associated with major tolerance concerns and off-label doxycycline/minocycline. By contrast, in March 2015, 1 mg/ $\mathrm{ml}$ ciclosporin (CSA) (Ikervis ${ }^{\circledR}$ ) was granted marketing authorisation by the European Commission $(\mathrm{EC})$ for "Treatment of severe keratitis in adult patients with DED, which has not improved despite treatment with tear substitutes."13

As an immunosuppressive agent belonging to the therapeutic class of ophthalmologicals, CSA blocks the release of proinflammatory cytokines and may exert an anti-inflammatory effect on ocular surface cells. ${ }^{14}$ In blocking "the vicious cycle of DED" this compound represents an important improvement in the treatment of the condition. $\mathrm{c}$

\section{Improving Delivery of Active Substances to the Ocular Surface}

\author{
Mourad Amrane
}

Santen SAS, Evry, France

The most common form of eye drops, which are the most straightforward and least expensive to develop, are aqueous solutions. However, these are associated with several drawbacks, mainly poor ocular penetration $(1 \%),{ }^{15}$ but also tolerability/safety and stability matters. Further, these solutions are limited to watersoluble drugs. Pharmaceutical manufacturers have therefore been faced with the difficulty of developing a formulation for topical administration to expand the range of potential active ingredients 
Figure 2: Novasorb ${ }^{\circledR}$ Cationic Nanoemulsions

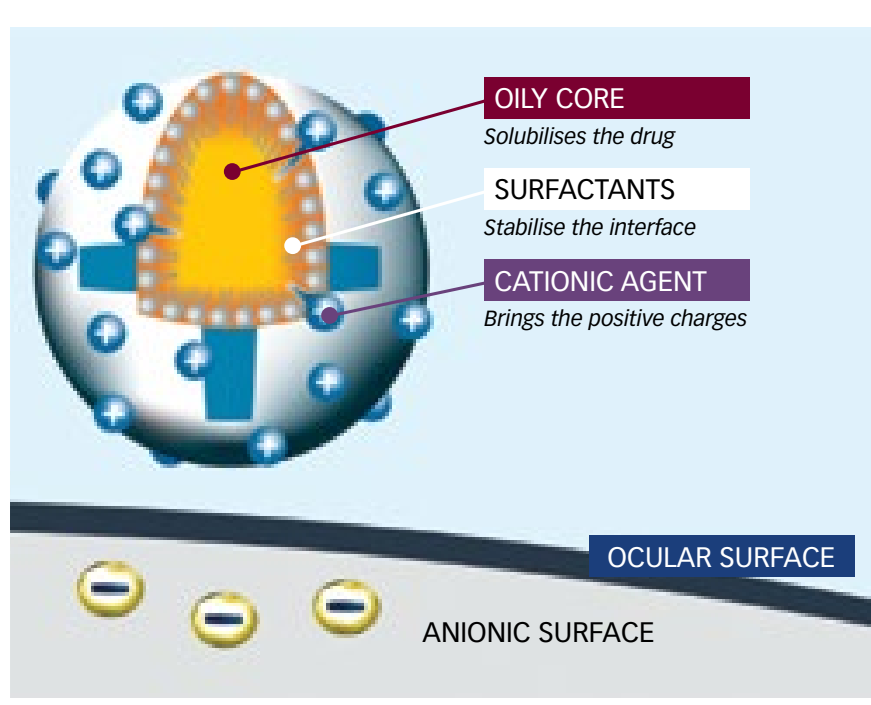

Adapted from: Lallemand et al., 2012.16

that remain longer at the ocular surface and provide sustained therapeutic concentrations.

Oil-in-water nanoemulsions enable the encapsulation of lipophilic drugs and offer improved stability as well as good tolerability and a high penetration rate (see Figure 2). ${ }^{16}$ Novasorb $^{\circledR}$ cationic nanoemulsions are characterised by a very small droplet size $(150 \mathrm{~nm})$, a zeta potential of up to $+40 \mathrm{mV}$, a viscosity equivalent to that of water $1.1 \mathrm{~mm}^{2} / \mathrm{s}$ and, crucially, a surface tension similar to that of human tears $(\sigma=41 \mathrm{mN} / \mathrm{m})$, which facilitates improved spreading over the eye. Further, Novasorb ${ }^{\circledR}$ cationic nanoemulsions are hypotonic to isotonic (180-300 mOsm/kg). Electrostatic interactions occur between the positively charged oil nanodroplets and the negatively charged ocular surface, leading to a prolonged residence time of the formulation on the ocular surface. In addition, the nanosize of oil droplets creates a huge contact surface with the corneal epithelial cells, thereby enhancing drug absorption. The contact angle $(\theta)$ (i.e. the angle in degrees between the formulation and the cornea), is very low compared with anionic emulsion, and hyaluronic acid formulation, which confirms the improved spreading of the product over the eye.

The multiple-dose safety of Novasorb ${ }^{\circledR}$ used as a drug vehicle to deliver CSA has been tested in a rabbit acute toxicity model. ${ }^{17}$ Three CSA formulations were tested: (i) a CSA formulation in cationic emulsion (CEm) (0.05 \% CSA-CEm); (ii) a $0.05 \%$ CSA-anionic emulsion (CSA-AEm); and (iii) $0.05 \%$ CSA-oil solution. Phosphate buffered saline (PBS) and $0.02 \%$ benzalkonium chloride (BAK) were used as the negative and toxic controls, respectively. The eye drops were applied to rabbit eyes 15 times at 5-minute intervals. The ocular surface structures were examined with a slit-lamp and by corneal in vivo confocal microscopy (IVCM) for detailed examination of corneal epithelium, stroma, limbus and conjunctiva-associated lymphoid tissue structure. The plotted scores of the IVCM scale ${ }^{17-19}$ analysis (see Figure 3) showed that CSA-CEm presented with the lowest toxicity, with toxicity patterns similar to PBS.
Figure 3: In Vivo Confocal Microscopy Scores of Three Different Ciclosporin Formulations in a Rabbit Model

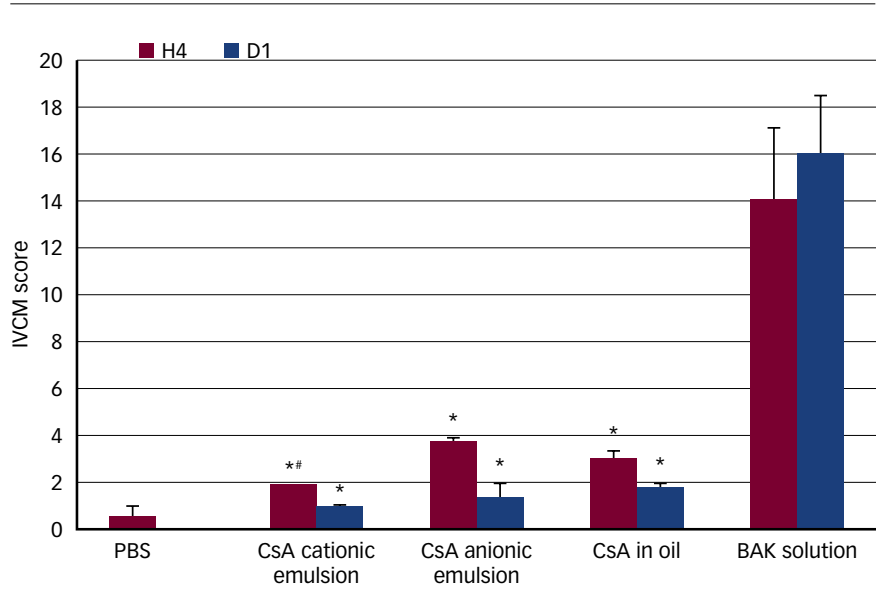

In vivo confocal microscopy (IVCM) scores were used to evaluate ocular surface toxicity profiles in four histological zones: superficial epithelium; basal epithelium; anterior stroma; and the limbus and conjunctiva. ${ }^{*} p \leq 0.03$ compared with phosphate buffered saline (PBS) and $p<0.0001$ compared with $0.02 \%$ benzalkonium chloride (BAK): $\#<0.05$ compared with ciclosporin-anionic emulsion (CSA-AEm). D1 = day $1 ; \mathrm{H} 4=$ hour 4 . Adapted from: Liang et al., 2012. ${ }^{17}$

\section{Figure 4: Single-dose Pharmacokinetic Comparison of Ciclosporin Cationic and Anionic Emulsions Showing Ciclosporin Concentrations in the Cornea Over Time}

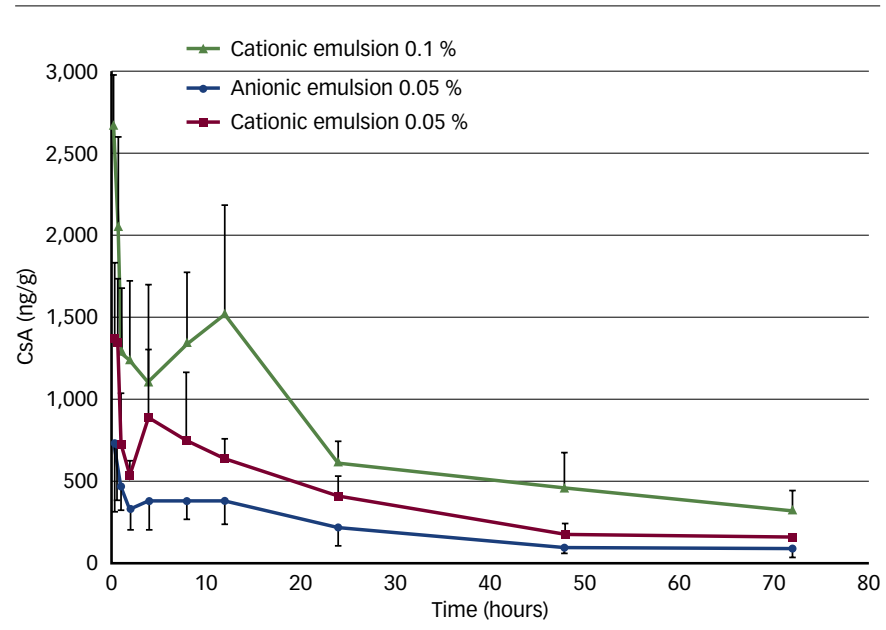

CSA = ciclosporin. Adapted from: Daull et al., 2013. ${ }^{19}$

Having demonstrated the good ocular tolerability of the CSA CEm, a single-dose pharmacokinetic study was conducted, using a rabbit model, to compare the corneal CSA bioavailability of CSA CEm $0.05 \%$ and CSA CEm $0.1 \%$ with anionic emulsion of CSA (0.05\%). CSA concentrations in corneal tissue were measured at 0-72 hour endpoints..$^{20}$ The CEms delivered higher CSA concentrations to the cornea (concentration maximum) than the anionic emulsion and had a superior exposition (area under the curve [AUC]) (see Figure 4). The $\mathrm{AUC}_{0-72} \mathrm{~h}$ for the CSA $0.1 \%$ and CSA $0.05 \%$ CEms were 51,817 ng.h/g and 26,703 ng.h/g, respectively, compared with only $14,333 \mathrm{ng} \cdot \mathrm{h} / \mathrm{g}$ for the CSA $0.05 \%$ anionic emulsion, indicating improved corneal bioavailability of the CSA CEms. The CSA CEm therefore appears to be a well tolerated and efficient eye drop to deliver CSA to the ocular surface. $\mathrm{c}$ 


\title{
Ikervis $^{\circledR} 1 \mathrm{mg} / \mathrm{ml}$ Ciclosporin - A New Therapeutic Option for the Treatment of Severe Keratitis in Dry Eye Disease
}

\author{
Andrea Leonardi
}

Department of Neuroscience, Ophthalmology, University of Padua, Italy

Ikervis $^{\circledR}$, an oil-in-water cationic eye drop emulsion based on the Novasorb ${ }^{\circledR}$ technology, is a single-dose product containing the active substance CSA $1 \mathrm{mg} / \mathrm{ml}$ (0.1\%), which has recently received approval from the European Commission (EC) for the treatment of severe keratitis in adult patients with DED, which has not improved despite treatment with tear substitutes. The recommended dose is one drop of Ikervis ${ }^{\circledR}$ once daily to be applied to the affected eye(s) at bedtime. ${ }^{13}$

\section{Methodology}

The SANSIKA study was carried out to investigate the efficacy and safety of Ikervis ${ }^{\circledast}$ (CSA $1 \mathrm{mg} / \mathrm{ml}$ eye drops) administered once daily in adult patients with severe keratitis in DED. SANSIKA was a multicentre, randomised, double-masked, two parallel arm, vehicle-controlled, 6-month phase III trial, which included a 6-month open-label treatment safety follow-up period (data on file). ${ }^{21}$ It was conducted across multiple sites across nine countries: Austria, two sites; Belgium, three sites; Czech Republic, one site; France, 19 sites; Germany, 13 sites; Italy, 12 sites; Spain, 14 sites; Sweden one site; and the UK, one site.

To be eligible for inclusion in the SANSIKA study, patients needed to have Grade 4 corneal fluorescein staining (CFS), as measured on a modified Oxford scale an Ocular Surface Disease Index (OSDI) questionnaire score $\geq 23$ at the screening and baseline visits and a Schirmer's test without anaesthesia scored $\geq 2 \mathrm{~mm} / 5 \mathrm{~min}$ and $<10 \mathrm{~mm} / 5 \mathrm{~min}$ at the screening visit (see Figure 5). After a 1-2 week wash-out period, patients were randomised to receive either CSA $1 \mathrm{mg} / \mathrm{ml}$ once daily or a once-daily vehicle emulsion. Primary efficacy and safety endpoints were measured at month 6 and subsequently all patients received CsA $1 \mathrm{mg} / \mathrm{ml}$ once daily for a 6-month open-label treatment safety follow-up period.

\section{Results}

At baseline, the CSA $(n=154)$ and vehicle groups $(n=91)$ were similar with respect to age (mean 60.8 and 62.1 years, respectively) and sex (81.8\% and $91.2 \%$ female, respectively) as well as the proportion of menopausal women specifically (70.6\% and $77.1 \%$, respectively) and the proportion of those with Sjögren's syndrome (37.7 \% and $37.4 \%$, respectively). Patients were also similar across a range of functional assessments, including CFS, OSDI, visual analogue scale (VAS), Schirmer's test, tear film break-up time and National Eye Institute-Visual Function Questionnaire (NEI-VFQ).

The primary endpoint was a composite measure whereby responders were identified if they showed $a \geq 2$ grade improvement in CFS and improvement in OSDI of $\geq 30 \%$. There was a higher proportion of responders in the CSA group (28.6\%) compared with vehicle $(23.1 \%)$ although the difference was not statistically significant (see Figure 6). Both the vehicle and the CSA group showed statistically significant improvement in OSDI scores at month 6 compared with baseline; however, the difference between the two groups was not statistically significant. On the planned secondary endpoint of CFS, patients who had received CsA $1 \mathrm{mg} / \mathrm{ml}$ showed significantly less staining at months

\section{Figure 5: SANSIKA Study Design}

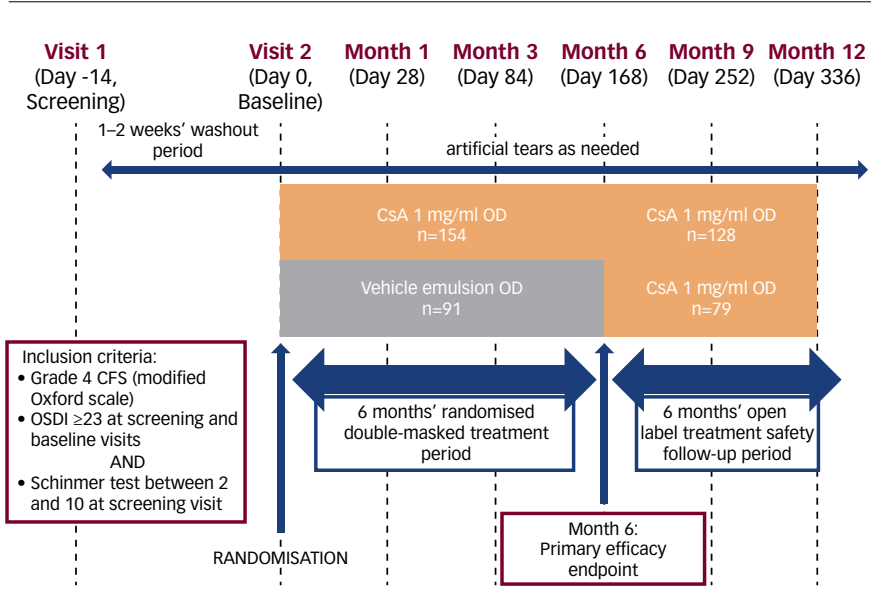

CFS = corneal fluorescein staining; CSA = ciclosporin; $O D=$ once daily; OSDI = Ocular surface Disease Index.

\section{Figure 6: Primary Composite Efficacy Outcomes}

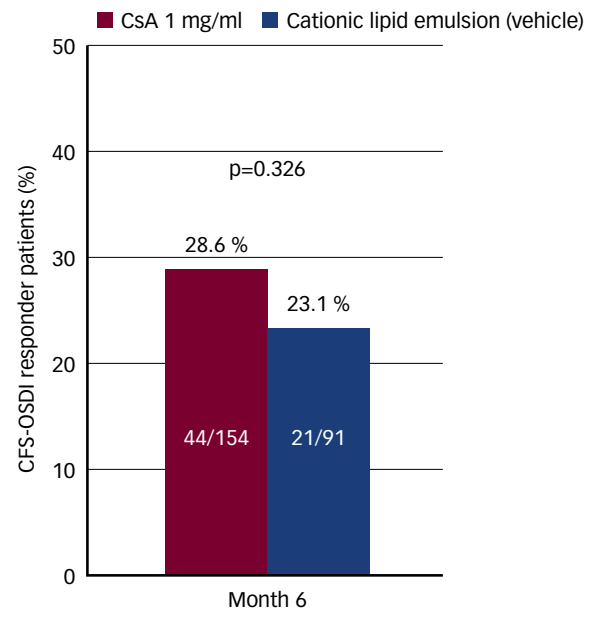

CSA = ciclosporin; $C F S=$ corneal fluorescein staining; OSDI = Ocular surface Disease Index .

3 and $6(p=0.024$ and $p=0.037)$ than those who had received vehicle (see Figure 7). The adjusted mean difference between the two groups in CFS was 0.35 at month $6 .{ }^{22}$ To better gauge the clinical relevance of this statistically significant difference, the mean difference can be converted to a ratio in the number of dots on the logarithmic modified Oxford scale. A difference of one grade on the modified Oxford scale corresponds to a multiplication of the number of dots by a factor of 3.16. ${ }^{23}$ The exception is the difference between a grade of zero (absence of dots) and Grade I (minimal, 10 dots on the cornea). Therefore, to switch from the mean difference to the ratio of the number of dots, the following formula was used:

$$
3.16^{\text {(mean difference) }}=3.16^{0.35}=1.50
$$


Figure 7: Secondary Outcomes - Corneal Fluorescein Staining - Modified Oxford Scale

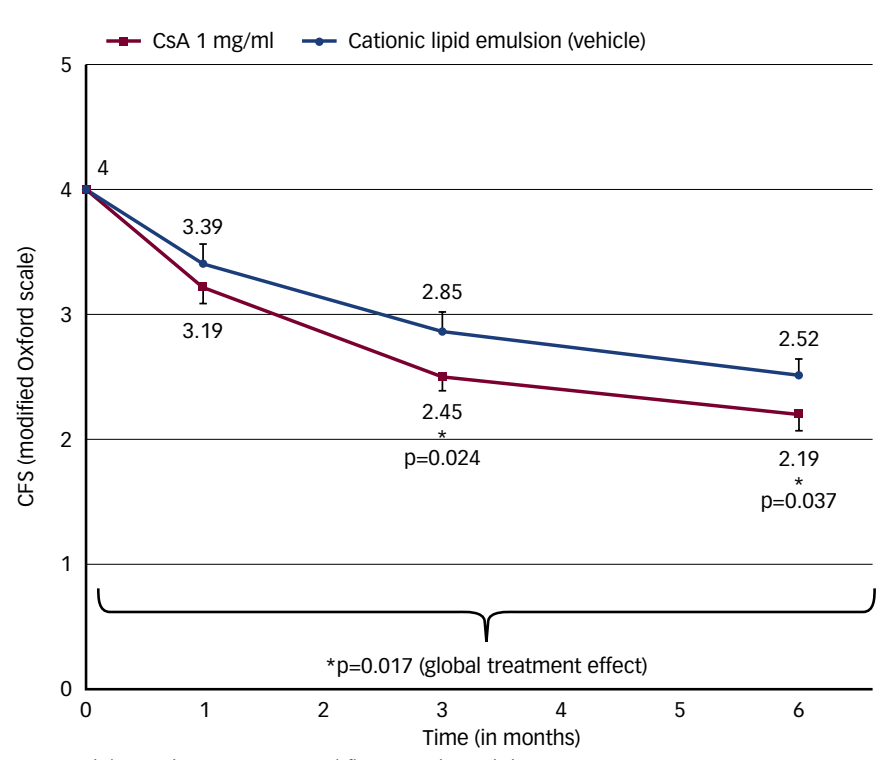

CSA $=$ ciclosporin CFS $=$ corneal fluorescein staining .

A ratio of 1.50 means that, at month 6 , the patients in the vehicle group have on average $50 \%$ more dots compared with the CSA CEm group, and, consequently, $50 \%$ more punctate corneal lesions.

Another method of analysing these CFS data is to compare the frequency distribution of CFS (see Figure 8) from baseline to that after 6 months' treatment. At baseline, all patients (100\%) exhibited Grade 4 CFS; only $20.5 \%$ remained at Grade 4 after 6 months. At month 6, a larger proportion of patients receiving CSA CEm (28.8\%) compared with vehicle (9.6\%) improved from Grade 4 to Grade 1 on the modified Oxford scale.

Measurement of the expression levels of the inflammatory biomarker human leukocyte antigen (HLA-DR), was a planned exploratory endpoint. Patients who had received CSA CEm showed significantly lower levels of expression of HLA-DR compared with patients who had received vehicle emulsion at Month 1 and Month 6 (see Figure 9).

There was no statistically significant difference in OSDI score over time for CsA $1 \mathrm{mg} / \mathrm{ml}$ versus vehicle. The mean change from baseline in the 100-point OSDI score was -13.6 with IKERVIS and -14.1 with vehicle at Month $6(p=0.858)$.

The main adverse event reported in the SANSIKA study was instillation site pain, of which there was a higher proportion in the Ikervis ${ }^{\circledR}$ group with $29.2 \%$ (mild: $16.9 \%$, moderate: $8.4 \%$ and severe: $3.9 \%$ ) compared with $8.9 \%$ with vehicle (mild: $4.4 \%$, moderate $2.2 \%$ and severe $2.2 \%$ ) (see Table 1) (data on file). Only seven patients had had detectable CSA blood levels (ranging from 0.105 to $1.27 \mathrm{ng} / \mathrm{mL}$ ) at Month 12 and the values were considered negligible in all cases (data on file) The systemic exposure profile seemed to be consistent with the profile observed until Month 6, whether the patient received CSA for 12 months or was switched to CSA after 6 months of receiving vehicle.

\section{SANSIKA Conclusions}

In the SANSIKA study, the severity of keratitis was reduced significantly at Month 3 and Month 6 with CsA compared with vehicle. The beneficial effect was maintained in the open phase of the study, from Month 6 and up to Month 12. Further, the proportion of CSA-

\section{Figure 8: Post Hoc Analysis - Corneal Fluorescein Staining Frequency Distribution}

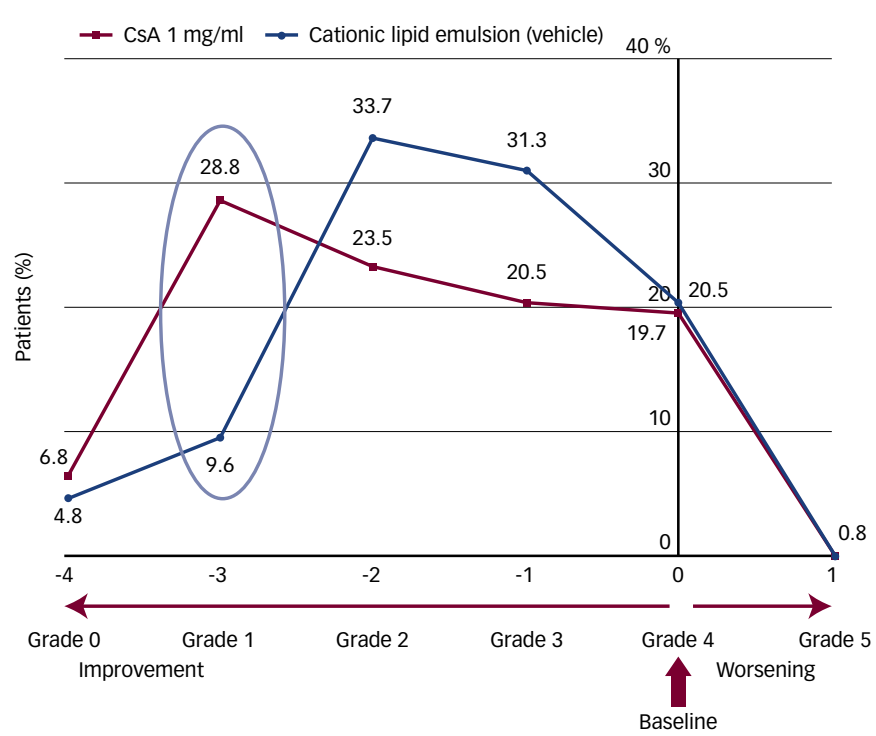

CSA $=$ ciclosporin

\section{Figure 9: Planned Exploratory Endpoint - Reduction in Human Leucocyte Antigen (HLA-DR) Expression}

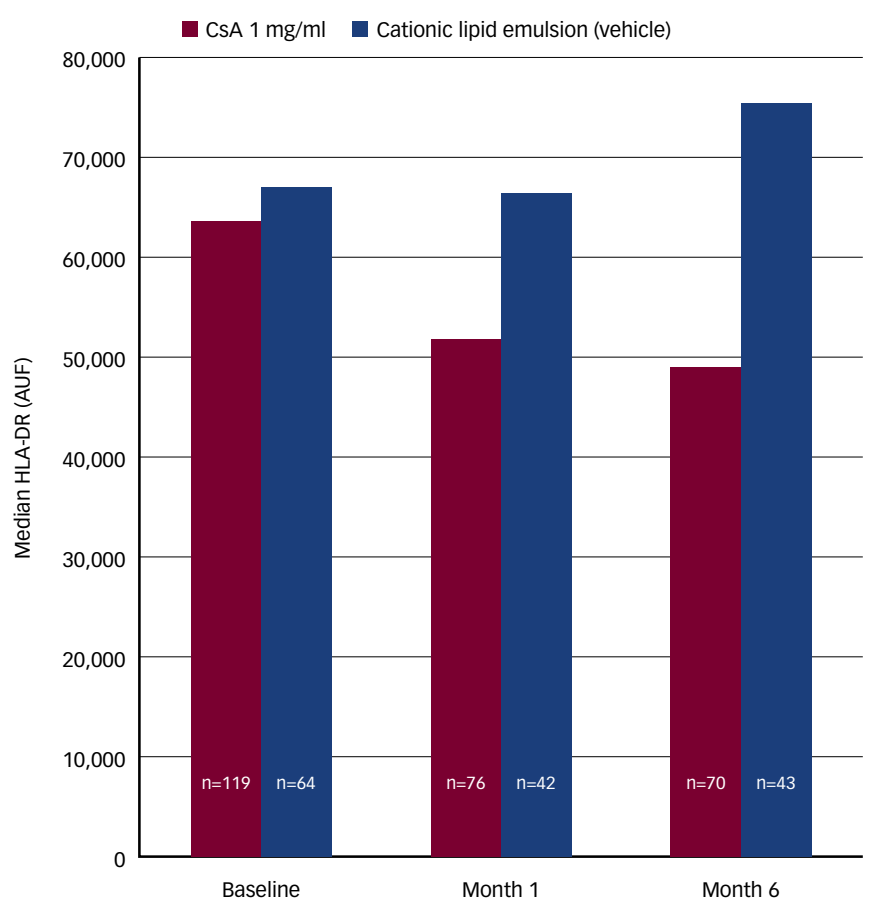

$A U F=$ arbitrary units of fluorescence; CSA = ciclosporin; HLA-DR = human leucocyte antigen-D related.

treated patients with a 3-grade improvement in CFS score at Month 6 (from grade 4 to 1 ) was $28.8 \%$, compared with $9.6 \%$ of vehicletreated subjects. A reduction in the ocular surface inflammation (HLA-DR expression) was observed at Month 6 in favour of CSA treatment over vehicle, providing additional support for the efficacy of CSA in the treatment of severe keratitis in adult patients with DED. Adverse events were infrequently reported with CSA or vehicle with the exception of instillation site pain, which a higher proportion of patients experienced in the CSA $1 \mathrm{mg} / \mathrm{ml}$ arm. Instillation site pain was mostly mild to moderate in severity and transitory in nature. $\square$ 
Table 1: Ocular-related Adverse Events by Severity in the SANSIKA Study Safety Population

\begin{tabular}{|c|c|c|c|c|c|c|}
\hline & \multicolumn{3}{|c|}{ CsA $1 \mathrm{mg} / \mathrm{ml}$} & \multicolumn{3}{|l|}{ Vehicle } \\
\hline & Mild n (\%) & Moderate n (\%) & Severe n (\%) & Mild n (\%) & Moderate n (\%) & Severe $\mathrm{n}(\%)$ \\
\hline \multicolumn{7}{|l|}{ Eye disorders } \\
\hline Blepharitis & $1(0.6)$ & 0 & $1(0.6)$ & $1(1.1)$ & 0 & 0 \\
\hline Conjunctival hyperaemia & $2(1.3)$ & 0 & 0 & 0 & 0 & 0 \\
\hline Dry eye & $1(0.6)$ & 0 & $1(0.6)$ & $1(1.1)$ & 0 & 0 \\
\hline Eye irritation & $1(0.6)$ & $1(0.6)$ & 0 & $1(1.1)$ & $1(1.1)$ & 0 \\
\hline Eye pruritus & 0 & $1(0.6)$ & 0 & $1(1.1)$ & 0 & 0 \\
\hline Eyelid oedema & $1(0.6)$ & 0 & $2(1.3)$ & 0 & 0 & 0 \\
\hline FBS & $1(0.6)$ & 0 & 0 & $1(1.1)$ & 0 & 0 \\
\hline Ocular hyperaemia & $1(0.6)$ & 0 & $1(0.6)$ & $1(1.1)$ & 0 & 0 \\
\hline Photophobia & 0 & $1(0.6)$ & 0 & $1(1.1)$ & 0 & $2(2.2)$ \\
\hline Visual acuity reduced & $1(0.6)$ & $1(0.6)$ & 0 & $1(1.1)$ & 0 & $1(1.1)$ \\
\hline \multicolumn{7}{|c|}{ General disorders and administration site conditions } \\
\hline Instillation site erythema & 0 & $2(1.3)$ & $1(0.6)$ & 0 & 0 & 0 \\
\hline Instillation site lacrimation & $1(0.6)$ & $1(0.6)$ & 0 & 0 & 0 & 0 \\
\hline Instillation site pain & $26(16.9)$ & $13(8.4)$ & $6(3.9)$ & $4(4.4)$ & $2(2.2)$ & $2(2.2)$ \\
\hline Instillation site pruritis & $1(0.6)$ & 0 & 0 & $1(1.1)$ & 0 & 0 \\
\hline
\end{tabular}

CSA = ciclosporin; FBS = foreign body sensation. SANSIKA Study. Data on File. ${ }^{21}$

\title{
How to Assess Ocular Surface Health
}

\author{
Gysbert van Setten
}

St Eriks Eye Hospital, Stockholm, Sweden

The correct assessment of ocular surface health is the cornerstone of understanding DED and initiating and maintaining optimal therapy. Many classic examination tools are available, including history taking, biomicroscopy, tear meniscus, Schirmer's test (I), ${ }^{24}$ CFS, conjunctival folds, tear film break-up time and Meibomian gland examination. These are useful to provide an orientation but can be overly time-consuming. Usually, an assessment begins with simple inspection, which can provide useful information, specifically with regards to epithelial changes, keratitis, inflammation, neovascularisation and the tear meniscus. However, adequate magnification of the ocular surface is required.

Models and investigations that constitute classic examination, such as those outlined in the 2007 Report of the International Dry Eye Workshop ${ }^{25}$ or The Report of the Tear Film \& Ocular Surface Society (TFOS) Workshop on Meibomian gland dysfunction, ${ }^{26}$ seldom uncover a simple history. Interpretation of the Schirmer's test (I) is critical, namely assigning the cut-off value to minimise false negatives and false positives and, hence the number of patients who remain undiagnosed. Other established diagnostic tests for DED include: CFS, hyper-osmolarity, Schirmer's test, impression cytology, filamentary keratitis, conjunctival staining, measuring visual acuity, Meibomian gland dysfunction or eyelid inflammation, blepharospasm, tear break-up time, aberrometry and confocal microscopy. Further tests include the measurement of inflammatory markers such as HLA-DR, matrix metallopeptidase (MMP)-9, cytokines and proteomic techniques used to identify protein biomarkers. It is clearly desirable to ensure that accurate diagnosis of DED is ubiquitously available and not only confined it to areas where these advanced diagnostic procedures are readily available. Diagnosis should be affordable, straightforward and quick. Neither is a fixation on assay-derived numbers helpful: a diagnostic gold standard has yet to be developed for DED and cut-off values should not be used in isolation but rather in the context of other assessments.
CFS is a simple, readily available tool that is straightforward in practice. However, it is challenging to use in connection with OSDI, in part because CFS is an expression of the condition and integrity of the ocular surface not just of surface damage. For example, in severe DED/Sjögren's Syndrome, as the severity and CFS increases the cornea sensitivity and hence OSDI sensitivity declines, emphasising the increasing importance of CFS. CFS is a cardinal sign of epithelial health, serving as an indicator of inflammation and MMP activity. It is important to remember that underlying surface damage, mediated by interleukins such as IL-17, is a sub-epithelial reaction, which involves induction of inflammation and raised MMP activity. In severe DED, camouflaging ocular surface diseases such as: microcystic epitheliopathy, corneal dystrophies, current erosions and post-laser treatment corneal scars may lead to a misinterpretation of visual changes. This underlines the challenges of diagnostic differentiation.

In summary, assessment of ocular surface health involves listening to the patient, the ability to apply low technological diagnostic tools, expertise in examination including the use of microscopy and an up-todate knowledge of current models of DED, and careful use of numeric results of automatic devices.

\section{Conclusion}

Ikervis ${ }^{\oplus}$, a stable cationic nanoemulsion of $1 \mathrm{mg} / \mathrm{ml}$ ciclosporin, based on the Novasorb ${ }^{\circledR}$ technology, is now available to treat severe keratitis in DED by blocking the vicious cycle process that is believed to be pivotal in the pathophysiology of DED. The SANSIKA study demonstrated a positive benefit-risk profile for CSA $1 \mathrm{mg} / \mathrm{ml}$ for the treatment of severe keratitis in DED. Assessment of ocular surface health is of crucial importance for the correct treatment choice and timing of treatment, and improved access to CSA is needed to enable patients with DED to benefit from this effective treatment. 
1. The definition and classification of dry eye disease: report of the Definition and Classification Subcommittee of the International Dry Eye WorkShop (2007), Ocul Surf, 2007;5:75-92 Bron AJ, Tomlinson A, Foulks GN, et al., Rethinking dry eye disease: a perspective on clinical implications, Ocul Surf. 2014;12(Suppl. 2):S1-31.

3. Lemp MA Crews LA Bron AJ, et al., Distribution of aqueousdeficient and evaporative dy eye in a clinic-based patient deficient and evaporative dry eye in a clinic-based patien

Lemp MA, Advances in understanding and managing dry eye dise MA, Advances in understanding and

Barabino S, Chen Y, Chauhan S, Dana R, Ocular surface Barabino S, Chen Y, Chauhan S, Dana R, Ocular surface
immunity: homeostatic mechanisms and their disruption in $\mathrm{dry}$ eye disease, Prog Retin Eye Res, 2012;31:271-85.

6. Stern ME, Schaumburg CS, Pflugfelder SC, Dry eye as a mucosa autoimmune disease, Int Rev Immunol, 2013;32:19-41. Ananthi S, Santhosh RS, Nila MV, et al., Comparative proteomics of human male and female tears by two-dimensional electrophoresis, Exp Eye Res, 2011;92:454-63.

8. Tong L, Zhou L, Beuerman RW, et al., Association of tear proteins with Meibomian gland disease and dry eye symptoms, $\mathrm{Br}$ Ophthalmol, 2011;95:848-52.

9. Wakamatsu TH, Dogru M, Ayako I, et al., Evaluation of lipid oxidative stress status and inflammation in atopic ocular oxidative stress status and inflammation in
surface disease, Mol Vis, 2010;16:2465-75.

10. Enriquez-de-Salamanca A, Castellanos E, Stern ME, et al., Tear cytokine and chemokine analysis and clinical correlations in evaporative-type dry eye disease, Mol Vis, 2010;16:862-73.

Baudouin C, A new approach for better comprehension of diseases of the ocular surface, J Fr Ophtalmol, 2007:30:239-46.

12. Sendrowski $D P$, Jaanus $S D$, Semes $L P$, Stern $M E$, Chapter 12: Antinflammatory Drugs. In: Bartlett JD, Jaanus SD, Clinical OCular Pharmacology, 5th ed St Louis, MO: Butterworth-Heinemann, 2007

13. European Medicines Agency. CHMP summary of positive opinion for lkervis 2012 Available at: $h$ ttp://mwwema. opinion for Kkenis 2012. Avallable at. htp./Muwwema. europa.eu/ema/index.jsp?curl=pages/medicines/human medicines/002066/smops/Positive/human_smop_000783. sp\&mid=WCOb01ac058001d127 (accessed 22 June 2015).

14. Donnenfeld E, Pflugfelder SC, Topical ophthalmic cyclosporine: pharmacology and clinical uses, Surv Ophthalmol, 2009;54:321 38.

15. Bucolo CM, Maltese A, Drago F, When nanotechnology meets the ocular surface, Expert Rev Ophthalmol, 2008;3:325-32.

16. Lallemand F, Daull P, Benita S, et al., Successfully improving ocular drug delivery using the cationic nanoemulsion, novasorb J Drug Deliv, 2012:2012:604204.

17. Liang H Baudouin C Daull Petal. Ocular safety of cationic emulsion of cyclosporine in an in vitro corneal wound-healing model and an acute cyclosporine in an in vitro corneal wound-healingm

18. Pauly A, Brignole-Baudouin F, Labbe A, et al., New tools for the evaluation of toxic ocular surface changes in the rat, Invest Ophthalmol Vis Sci, 2007;48:5473-83.

19. Liang H, Baudouin C, Pauly A, Brignole-Baudouin F, Conjunctival and corneal reactions in rabbits following short- and repeated exposure to preservative-free tafluprost, commercially available latanoprost and $0.02 \%$ benzalkonium chloride, Br I Ophthalmol 2008:92:1275-82

20. Daull P, Lallemand F, Philips B, et al., Distribution of cyclosporine A in ocular tissues after topical administration of cyclosporine A cationic emulsions to pigmented rabbits, Cornea, 2013;32:345-54.

21. SANSIKA safety and tolerability results. Data on file, Santen, March 2016. Job code: STN 0318 IKV 00042

22. Leonardi A, Van Setten $G$, Amrane M, et al., Efficacy and safety of $0.1 \%$ cyclosporine A cationic emulsion in the treatment of severe dry eye disease: a multicenter randomized trial, Eur J Ophthalmol, 2016 Apr 7:0. doi: 10.5301/ejo.5000779.

23 Bron AJ, Evans VE, Smith JA, Grading of corneal and conjunctival staining in the context of other dry eye tests, Cornea, 2003;22:640-50

24. Stevens S, Schirmer's test, Community Eye Health, 2011;24:45.

25. Research in dry eye: report of the Research Subcommittee of the International Dry Eye WorkShop (2007), Ocul Surf, 2007:5:179-93.

26. Nichols KK, Foulks GN, Bron AJ, et al., The international workshop on meibomian gland dysfunction: executive summary, Invest Ophthalmol Vis Sci, 2011;52:1922-9.

\section{Abbreviated Prescribing Information}

Please refer to the product Summary of Product Characteristics for full details.

Product name: IKERVIS ${ }^{\circledast} 1 \mathrm{mg} / \mathrm{mL}$ eye drops, emulsion

Composition: $1 \mathrm{ml}$ of emulsion contains $1 \mathrm{mg}$ of ciclosporin and $0.05 \mathrm{mg}$ cetalkonium chloride as an excipient. Please refer to the Summary of Product Characteristics (SmPC) for a full list of excipients.

Indication: Treatment of severe keratitis in adult patients with dry eye disease, which has not improved despite treatment with tear substitutes.

Dosage and administration: IKERVIS ${ }^{\circledR}$ treatment must be initiated by an ophthalmologist or a healthcare professional qualified in ophthalmology. The recommended dose is one drop of IKERVIS ${ }^{\circledR}$ once daily to be applied to the affected eye(s) at bedtime. Response to treatment should be reassessed at least every 6 months. To reduce systemic absorption, advise patients to use nasolacrimal occlusion and to close the eyelids for 2 minutes after instillation. If more than one topical ophthalmic product is used, 15 minutes should separate their administration. IKERVIS should be administered last.

Contraindications: Hypersensitivity to any of the ingredients. Active or suspected ocular or peri-ocular infection.

Warnings and precautions: Use with caution in patients with a history of ocular herpes. Contact lenses: Patients wearing contact lenses have not been studied. Monitor carefully inpatients with severe keratitis. Contact lenses should be removed before instillation of the eye drops at bedtime and may be reinserted at wake-up time. Concomitant therapy: Use with caution in patients with glaucoma, especially in those receiving concomitant beta-blockers which are known to decrease tear secretion. Immune system effects: Medicinal products which affect the immune system, including ciclosporin, may affect host defences against infections and malignancies Contains cetalkonium chloride which may cause eye irritation.

Interactions with other medicinal products: Coadministration with eye-drops containing corticosteroids may potentiate effects on the immune system.

Pregnancy and breast feeding: Not recommended in women of childbearing potential not using effective contraception or during pregnancy unless the potential benefit to the mother outweighs the potential risk to the foetus. Benefits of treatment must be weighed against the benefits of breast feeding.

Driving and using machines: Moderate influence on the ability to drive and use machines. If blurred vision occurs on instillation, the patient should be advised to not drive or use machines until their vision has cleared.

Undesirable effects: Consult SMPC for full details. The most common adverse reactions in clinical studies were eye pain, eye irritation, lacrimation, ocular hyperaemia and eyelid erythema. Patients receiving immunosuppressive therapies including ciclosporin, are at an increased risk of infections.

Special precautions for storage: DO not freeze. After opening of the aluminium pouches, the single-dose containers should be kept in the pouches in order to protect from light and avoid evaporation. Discardany opened individual single-dose container with any remaining emulsion immediately after use.

Package quantities and basic NHS cost: $30 \times 0.3 \mathrm{ml}$ single-dose containers $\mathrm{f} 72.00$.

Product licence holder: Santen Oy, Niittyhaankatu 20, 33720 Tampere, Finland (PL 16058/0012) (EU/1/15/990/001 \& 002)

Date of authorisation: March 2015

Legal category: POM

Date of last revision of prescribing information: 02/09/2015

IKERVIS ${ }^{\circledR}$ is a registered trademark of Santen Pharmaceuticals Co., Ltd.

Job code: STN 0617 IKV 00004b

Adverse events should be reported. Reporting forms and information can be found at www.mhra.gov.uk/yellowcard. Adverse events should also be reported to Santen UK Limited (E: medinfo@santen. co.uk or telephone: 0345075 4863). 